\title{
eJRIEPS
}

Ejournal de la recherche sur l'intervention en éducation physique et sport

$22 \mid 2011$

Varia

\section{Auto-évaluation d'élèves et prédiction de l'évaluation de l'éducateur physique à leur égard : incidences sur leurs comportements en éducation physique}

Denis Martel, Jocelyn Gagnon et Paul Godbout

\section{(2) OpenEdition}

Journals

Édition électronique

URL : http://journals.openedition.org/ejrieps/4721

DOI : 10.4000/ejrieps.4721

ISSN : 2105-0821

Éditeur

ELLIADD

Référence électronique

Denis Martel, Jocelyn Gagnon et Paul Godbout, « Auto-évaluation d'élèves et prédiction de l'évaluation de l'éducateur physique à leur égard : incidences sur leurs comportements en éducation physique », eJRIEPS [En ligne], 22 | 2011, mis en ligne le 01 janvier 2011, consulté le 21 décembre 2020. URL http://journals.openedition.org/ejrieps/4721 ; DOI : https://doi.org/10.4000/ejrieps.4721

La revue eJRIEPS est mise à disposition selon les termes de la Creative Commons Attribution 4.0 International License. 
Auto-évaluation d'élèves et prédiction de l'évaluation de l'éducateur physique à leur égard : incidences sur leurs comportements en éducation physique

Denis Martel, Jocelyn Gagnon \& Paul Godbout

Département d'éducation physique, Université Laval, Québec, Canada

\section{Résumé}

Les objectifs de cette étude étaient (1) d'analyser l'auto-évaluation d'élèves et leur prédiction de l'évaluation de leur enseignant d'éducation physique et (2) de vérifier leur relation avec les comportements qu'ils disent adopter en classe d'éducation physique. Un total de 891 élèves (418 filles, 473 garçons) et huit enseignants ont participé à l'étude. L'écart entre l'auto-évaluation des élèves et leur prédiction de l'évaluation de leur enseignant a été mesuré pour leur niveau performance, leur niveau de discipline et l'intensité de leurs efforts. Les résultats montrent que plus les élèves s'estiment sousévalués par leur enseignant sur ces trois dimensions, moins ils tendent à rapporter l'adoption de comportements positifs durant les cours d'éducation physique, les garçons signalant encore moins de comportements positifs. Ces résultats suggèrent que la perception qu'ont les élèves de ce que leur enseignant pense d'eux a un impact sur ce qui se passe en classe d'éducation physique.

\section{Introduction}

Cette étude s'inscrit dans le courant de recherche qui a pour but de caractériser les perceptions des élèves sur ce qu'ils pensent au sujet des conditions d'apprentissage qui prévalent dans leur cours d'éducation physique. La pertinence de ce type d'études repose sur le fait que ce que vivent et ressentent les élèves influence grandement leur conduite et leur rendement scolaire (Dyson, 1995). Lee et Solmon (2005) ont plus récemment bien résumé l'importance d'étudier ces variables associées aux élèves :

"Les élèves se présentent aux cours avec des savoirs, des expériences, des valeurs et des croyances préalables qui font office de lentilles ou de filtres au travers desquels ils perçoivent leur milieu d'apprentissage. En se fondant sur leurs perceptions d'événements qui se produisent en cours d'EPS, ils donnent un sens à leurs expériences et interprètent les stimuli d'apprentissage. Ils prennent des décisions quant à leurs interactions en classe, décidant ou non de s'engager dans les situations d'apprentissage, décidant combien d'effort y consacrer, et décidant 


\section{eJRIEPS 22 janvier 2011}

de persister ou non en cas de difficultés. De ce point de vue, il importe d'investiguer les activités cognitives des élèves afin d'apprendre comment les enseignants peuvent construire le climat qui encouragera ces élèves à penser et à agir de façons favorables à leur apprentissage ». (p. S-114, traduction libre)

De nombreuses études ont considéré les perceptions et comportements des élèves en rapport avec les attentes et comportements de leurs enseignants. Un thème de recherche particulièrement étudié a été celui de l'effet Pygmalion (pour une revue détaillée, consulter Trouilloud et Sarrazin, 2003). Tel qu'illustré à la Figure 1, le modèle de Martinek (1991) résume le processus de l'effet Pygmalion de la façon suivante:

« a) les enseignants développent des attentes envers leurs élèves à partir de diverses caractéristiques d'un élève ; b) à partir de ces perceptions, l'enseignant développe certaines attentes quant aux performances futures de l'élève ; c) ces attentes peuvent influencer la quantité et la qualité des interactions entre l'enseignant et l'élève ; d) l'élève perçoit et interprète ces interactions et peut performer ou ne pas performer d'une manière conforme aux attentes de l'enseignant ». (p. 60, traduction libre)

Plus récemment, Trouilloud, Sarrazin, Martinek et Guillet (2002) précisent que le processus de l'effet Pygmalion, quel que soit le modèle théorique utilisé dans six ouvrages cités, comporte trois phases:

«(1) les enseignants développent des attentes quant aux réalisations futures de leurs élèves, (2) ils traitent différemment leurs élèves (d'un point de vue qualitatif et quantitatif) en fonction de ces attentes, et (3) ce traitement différencié influence directement, ou indirectement via des variables motivationnelles ou cognitives (telles les perceptions que les élèves ont d'eux-mêmes), les performances des élèves ». (p. 592, traduction libre) 


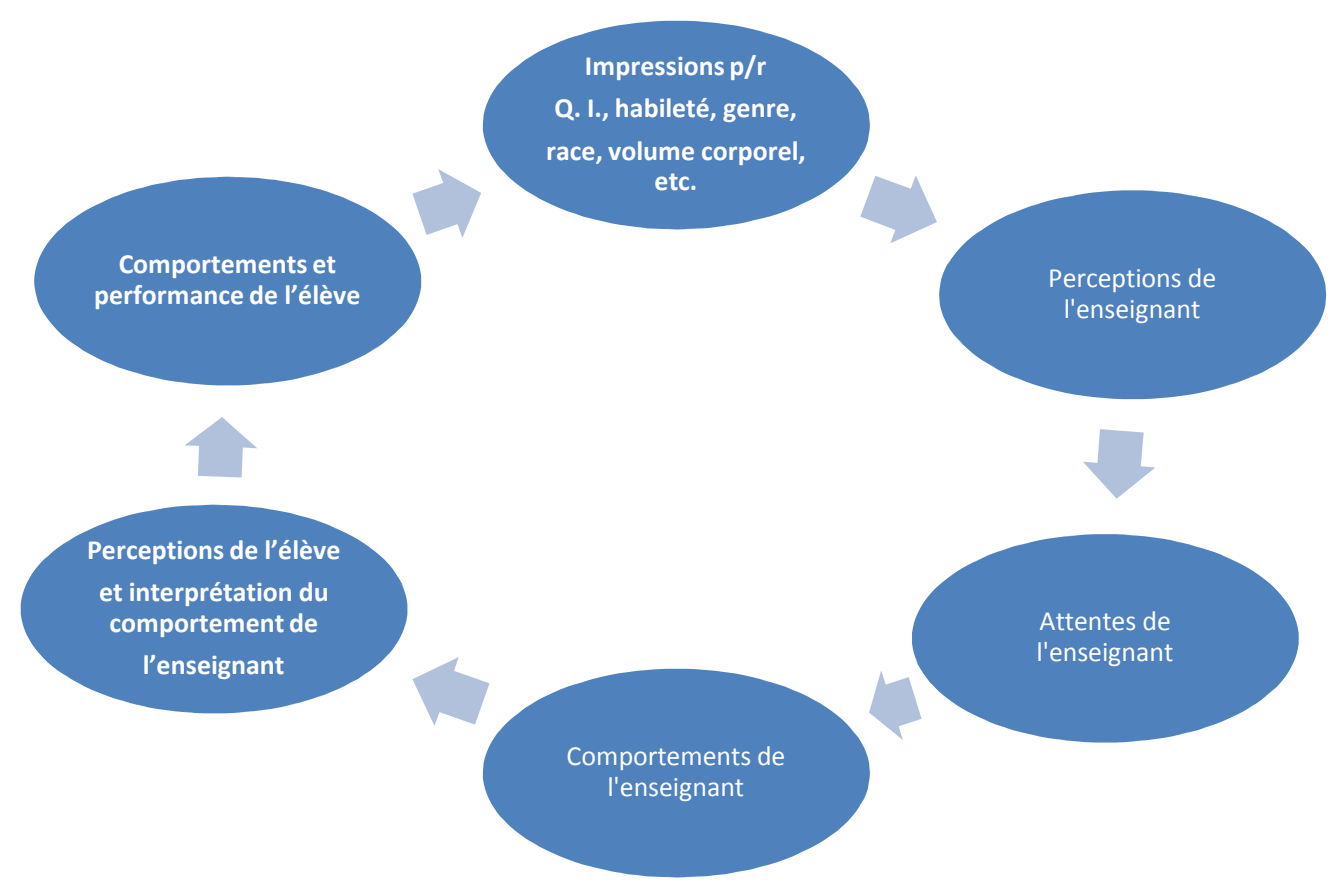

Figure 1. Le modèle des attentes de l'enseignant en éducation physique et sportive (Martinek, 1991)

Bien que cette étude ne porte pas directement sur l'effet Pygmalion, elle est fortement inspirée de recherches réalisées en éducation physique en rapport avec ce phénomène. Plus précisément, elle traite des perceptions qu'ont les élèves des comportements de leur enseignant à leur égard et de leurs réactions positives ou négatives à ces comportements d'enseignants. Selon Martinek (1988), cette variable s'avère critique pour le processus enseignement-apprentissage dans la mesure où les perceptions qu'ont les élèves des comportements de leur enseignant, et particulièrement l'interprétation qu'ils en font, ont une forte influence sur leurs propres comportements et leur performance. En fait, la signification que les élèves prêtent aux comportements de leur enseignant agirait comme un filtre entre les actions de celui-ci et leur impact sur eux (Fraser, 1994). Essentiellement, cela signifie que les élèves peuvent interpréter différemment un même comportement d'enseignant (Good, 1987) et réagir de diverses façons à ce comportement (Brophy, 1983). Martinek (1981a) explique ce phénomène par l'hypothèse qu'il existe une grande variabilité dans les interprétations que font les élèves des comportements de leur enseignant.

1. 1. Perceptions des élèves des comportements de leur enseignant Les résultats des quelques études qui ont décrit les perceptions des élèves en rapport avec les comportements des enseignants indiquent que les élèves sont conscients des différents traitements accordés à ceux qui éprouvent des difficultés à l'école par rapport à 


\section{eJRIEPS 22 janvier 2011}

ceux qui réussissent facilement (Babad, Bernieri et Rosenthal, 1991; Brattesani et Weinstein, 1980; Brattesani, Weinstein et Marshall, 1984; Marshall et Weinstein, 1982; Weinstein, 1983; Weinstein, Marshall, Brattesani et Middlestadt, 1982; Weinstein, Marshall, Sharp et Botkin, 1987). De façon plus spécifique, les études de Weinstein et ses collaborateurs ont permis de constater que les élèves estiment que ceux qui sont moins performants reçoivent plus de feed-back négatifs et de directives, qu'ils ont plus de travail à faire et sont soumis à plus de règlements que ceux qui obtiennent de bons résultats. À l'inverse, ils perçoivent que ceux qui ont du succès jouissent d'attentes plus élevées et profitent d'une plus grande liberté de choix. Ces impressions étaient partagées par l'ensemble des élèves indépendamment de la nature des attentes dont ils faisaient l'objet. De plus, les élèves perçoivent les préjugés des enseignants indépendamment des méthodes d'enseignement qu'ils privilégient. Enfin, les élèves de six ou sept ans perçoivent aussi bien les iniquités qui surviennent dans leur classe que les élèves âgés de 10 et 11 ans. II semble donc que les élèves peuvent percevoir les traitements particuliers dont font l'objet leurs pairs marqués d'attentes faibles ou élevées. II faut toutefois mentionner que dans ces études, les élèves devaient se prononcer sur les comportements de leur enseignant envers les autres élèves et non sur la nature de ses comportements à leur égard.

Les recherches en éducation physique sur les perceptions des élèves au sujet des comportements de leur enseignant sont tout aussi rares que celles en enseignement en général. Ces quelques recherches (Daniels, 1983; Martinek, 1988; Martinek et Karper 1986; Morency, 1990; Mros, 1990) ont toutefois permis l'émergence de conclusions intéressantes sur ce que pensent les élèves de la conduite de leur éducateur physique. Ceux-ci perçoivent que l'éducateur physique n'agit pas de la même façon avec tous les élèves de leur groupe (Martinek, 1988, Morency, 1990; Mros, 1990). Plus particulièrement, les élèves faisant l'objet d'attentes élevées se disent plus souvent félicités que réprimandés (Martinek 1988) et estiment être davantage supportés que les élèves moins habiles (Martinek et Karper, 1986). De plus, les garçons affirment que leur éducateur physique les encourage plus souvent qu'il n'encourage les filles (Daniels, 1983). Enfin, l'interprétation que font les élèves des comportements de l'éducateur physique à leur égard n'est pas la même pour tous. En effet, les élèves qui bénéficient d'attentes élevées attribuent leurs punitions aux traits de caractère de leur éducateur physique (Martinek, 1988). Ils croient que les encouragements qu'ils reçoivent sont dus à leurs habiletés personnelles et que les réprimandes sont provoquées par leur manque d'effort (Mros, 1990). Les élèves qui font l'objet d'attentes faibles sont plutôt portés à s'identifier comme 


\section{eJRIEPS 22 janvier 2011}

la cause de la plupart des comportements que l'éducateur physique adopte à leur égard (Martinek, 1988). Ils estiment qu'ils sont réprimandés surtout parce qu’ils sont indisciplinés (Martinek, 1988) ou qu'ils sont peu habiles (Mros, 1990).

Par ailleurs, les recherches portant sur l'auto-perception des élèves ont accordé beaucoup d'attention à la notion de compétence, que ce soit en sport ou en éducation physique, et à la source d'information à laquelle les élèves tendent à se référer (Halliburton \& Weiss, 2002 ; McKiddie \& Maynard, 1997 ; Weiss \& Amorose, 2005 ; Weiss, Ebbeck, \& Horn, 1997). Bien que ces recherches aient souvent été conduites avec des jeunes garçons et filles (niveau primaire et $5^{\text {ième }}$ et $6^{\text {ième }}$ du collège), elles n'ont pas discuté ou rapporté de différences entre les deux sexes. En France, des recherches conduites auprès d'étudiants du collège ont révélé que les garçons affirment recevoir plus de rétroactions négatives mais se sentent plus compétents que les filles en éducation physique (Nicaise, 2005; Nicaise, Cogérino, Bois, \& Amorose, 2006). Pour sa part, Lentillon (2005) a conclu que des garçons de niveau collège se sentent davantage sous-évalués que les filles en éducation physique bien qu'ils obtiennent de meilleures notes. Enfin, une étude réalisée auprès d'adolescents et adolescentes de niveau collégial a révélé que les filles percevaient recevoir plus fréquemment de l'encouragement et des informations techniques alors que les garçons avaient le sentiment que leur enseignant les critiquait davantage et était plus susceptible d'ignorer leurs erreurs (Nicaise, Bois, Fairclough, Amorose, et Cogérino, 2007).

1. 2. Écart entre l'auto-évaluation des élèves et leur perception de l'évaluation de l'enseignant : notions de concordance et discordance

Un programme de recherche conduit par Gagnon et ses collaborateurs s'est intéressé à la comparaison entre la perception que les élèves ont d'eux-mêmes (auto-évaluation) et leur prédiction de l'évaluation de l'enseignant d'EPS à leur égard. Cette prédiction de l'évaluation de l'éducateur physique par les élèves repose sur leur interprétation des comportements de ce dernier à leur égard lors des cours d'éducation physique. Les travaux de recherche ont permis d'identifier trois types d'écart entre l'auto-évaluation des élèves et leur prédiction de l'évaluation de l'enseignant et de quantifier la prévalence de ces écarts (Gagnon, 1992 ; Gagnon, Martel, Michaud, Valois, \& Gagné, 2005; Julien, 2003; Pelletier-Murphy, 2004). La présence ou l'absence d'un écart permet de déterminer le niveau de discordance, ou de concordance, entre l'image que les élèves ont d'eux-mêmes et l'image qu'ils croient que leur enseignant a de chacun d'eux. Plus précisément, une concordance signifie que l'élève se sent évalué à sa juste valeur, son auto-évaluation étant identique à son estimation de l'évaluation de l'enseignant. Dans tous les autres cas, 


\section{eJRIEPS 22 janvier 2011}

il y a discordance, c'est-à-dire que l'auto-évaluation de l'élève diffère de son estimation de l'évaluation que lui accorderait l'enseignant. Une discordance positive signifie que l'élève se sent surévalué ; à l'inverse, il y a discordance négative lorsque l'élève se sent sousévalué.

Les résultats de deux recherches réalisées avec des élèves de $5^{\text {ième }}$ et $6^{\text {ième }}$ année du primaire (10-11 ans) montrent que seulement le tiers d'entre eux estiment que leur enseignant d'EPS les évalue correctement sur chacune de trois dimensions, à savoir leur niveau de performance, de discipline et d'effort. Les autres pensent, à des degrés divers, que leur enseignant a une idée plus ou moins juste de leur niveau eu égard aux trois dimensions susmentionnées (Julien, 2003 ; Pelletier-Murphy, 2004). De plus, lorsque l'on considère les trois dimensions séparément, quelque $25 \%$ des élèves s'estiment sous-évalués sur leur niveau de performance, environ $20 \%$ croient qu'ils sont sous-évalués quant à leur niveau de discipline, et enfin près de $35 \%$ prétendent que leurs efforts sont sous-évalués (Gagnon et al., 2005 ; Pelletier-Murphy, 2004).

Sous un autre angle, la perception qu'ont les élèves du comportement de l'enseignant à leur endroit varie selon le type de discordance et sa magnitude (Julien, 2003 ; PelletierMurphy, 2004). Plus précisément, les élèves qui signalent être correctement évalués ou légèrement surévalués semblent très satisfait de leur sort et se sentent encouragés à adopter et maintenir une attitude positive durant les cours d'EPS. À l'inverse, les élèves qui s'estiment fortement sous-évalués sont insatisfaits de leur sort et indiquent que leur insatisfaction les amène à adopter des comportements inappropriés. Enfin, pour les cas où sont perçues une légère sous-estimation ou une forte surestimation, on retrouve un peu de tout: élèves satisfaits, insatisfaits, et d'autres parfois satisfaits et parfois insatisfaits.

\section{3. Les questions de recherche}

Dans l'ensemble, les travaux antérieurs de l'équipe de recherche de Gagnon et ses collaborateurs ont porté presque exclusivement sur les types de discordance et leur amplitude pour chacune des trois dimensions considérées séparément. Dans la présente étude, les perceptions des élèves sont analysées en fonction de profils de discordance qui résultent de la combinaison des types de discordance sur l'ensemble des trois dimensions pour lesquelles les perceptions des élèves ont été mesurées (performance, discipline et effort). Les différents types de profils sont présentés à la sous-section 2.4.2 de la section Méthodologie.

Les données de recherches ont été analysées en vue de répondre aux trois questions suivantes: 


\section{eJRIEPS 22 janvier 2011}

1- Quels sont, chez des élèves de $5^{\text {ième }}$ et $6^{\text {ième }}$ années du primaire (garçons et filles), les profils de discordance entre leur auto-évaluation et leur perception de l'évaluation de leur enseignant en ce qui a trait à leur niveau de performance, de discipline et d'effort durant les cours d'EPS ? Y a-t-il des différences entre garçons et filles ?

2- Y a-t-il une relation entre les profils de discordance révélés par les élèves de chaque sexe et les comportements qu'ils disent adopter durant les cours d'EPS ?

3- $Y$ a-t-il une relation, pour chacune des trois dimensions, entre l'amplitude de la discordance perçue par les élèves de chaque sexe et les comportements qu'ils disent adopter durant les cours d'EPS ?

\section{Méthodologie}

\section{1. Les participants}

Un total de 891 élèves (418 filles, 473 garçons), issus de 35 classes régulières de $5^{\mathrm{e}}$ $(\mathrm{N}=21)$ et $6^{\mathrm{e}}(\mathrm{N}=14)$ années d'écoles primaires des régions urbaines de Québec et TroisRivières, ont complété un questionnaire. L'âge de ces élèves variait entre 10 et 11 ans. Huit éducateurs physiques ont agi à titre d'intermédiaires pour faciliter les rencontres entre les chercheurs et les élèves.

\section{2. Le questionnaire}

Un questionnaire a été utilisé afin de recueillir les perceptions des élèves en rapport avec leur performance en éducation physique, leur niveau de discipline et l'intensité de leurs efforts. Ce questionnaire est issu d'études québécoises antérieures (Martel, Gagnon, Grenier, Pelletier-Murphy, \& Dumont, 1999; Martel, Pelletier-Murphy, \& Gagnon, 1999) et le choix des trois dimensions (performance, discipline, effort) repose sur le fait qu'elles sont les variables les plus déterminantes dans le développement des attentes des enseignants (Dostie, 1996). Par ailleurs, la liste des comportements d'éducateurs physiques ( $\mathrm{N}=20$ ) provient d'une étude qui a permis d'identifier les comportements d'enseignement favorables $(\mathrm{N}=10)$ et défavorables $(\mathrm{N}=10)$ qui constituent, selon des élèves de $5^{\mathrm{e}}$ et $6^{\mathrm{e}}$ années, les meilleurs indices de la nature des attentes d'un éducateur physique (Martel, Gagnon, Pelletier-Murphy, et al., 1999). Quant aux listes des sentiments $(\mathrm{N}=17)$ et des réactions des élèves $(\mathrm{N}=18)$, elles sont tirées d'une étude pilote réalisée auprès de cent élèves de $5^{\mathrm{e}}$ et $6^{\mathrm{e}}$ années du primaire. Lors de cette étude, les élèves n'avaient aucun choix de réponse (question ouverte) pour répondre aux questions «Qu'est-ce que cela te fait ?» et «Comment réagis-tu lorsque cela se produit?». L'analyse inductive de leurs réponses (600 énoncés de sentiments, 600 énoncés de réactions), sur 


\section{eJRIEPS 22 janvier 2011}

la base d'un consensus entre analystes, a permis la création des listes de 17 sentiments et de 18 réactions d'élèves ainsi que leur catégorisation (sentiment positif ou négatif; réaction positive ou négative) non indiquée dans le questionnaire. Les nouveaux comportements d'enseignants, sentiments et réactions identifiés lors de la présente étude ont également été catégorisés (positif ou négatif) sur la base d'un consensus interanalystes.

\section{3. Les procédures de passation du questionnaire}

La passation du questionnaire fût réalisée auprès de tous les élèves vers la fin de l'année scolaire dans la classe du titulaire et ce, en l'absence de ce dernier et de l'éducateur physique. Deux chercheurs supervisaient les élèves afin de s'assurer qu'ils comprennent adéquatement les questions et qu'ils inscrivent leurs réponses aux endroits appropriés sur le questionnaire. Cette procédure s'était avérée très pertinente lors d'études antérieures réalisées avec des élèves du même niveau scolaire (Martel, Gagnon, Grenier, et al., 1999 ; Martel, Pelletier-Murphy et al., 1999).

Concrètement, les élèves devaient d'abord inscrire leur nom sur le questionnaire de même que celui de leur éducateur physique. Les élèves étaient informés de la nécessité d'indiquer leur nom afin d'éviter toute confusion et que ce dernier serait transposé en numéro lors de l'analyse des résultats. Les chercheurs prenaient également soin d'indiquer aux élèves que la confidentialité de leurs réponses serait assurée. Par la suite, chaque élève complétait individuellement le questionnaire selon une procédure systématique illustrée ci-après à partir de la section du questionnaire relative à la dimension performance (Tableau I). Lors de cette procédure, chaque élève devait : 1) encercler sur l'échelle d'appréciation graduée de 1 à 5 la cote qui selon lui correspondait à sa performance en éducation physique (Question 1.1); 2) encercler sur l'échelle d'appréciation graduée de 1 à 5 la cote qu'il croyait que son éducateur physique lui donnerait sur sa performance en éducation physique (Question 1.2); 3) encercler (couleur bleue) le numéro du comportement qui constituait le meilleur indice pour justifier la cote qu'il croyait que son éducateur physique lui donnerait sur sa performance (Question 2.1); 4) encercler (couleur bleue) le numéro du sentiment ressenti lors de l'adoption de ce comportement par l'éducateur physique à son égard (Question 2.2); 5) encercler (couleur bleue) le numéro de sa façon de réagir face à ce comportement de l'éducateur physique à son égard (Question 2.3). Les étapes 4 et 5 étaient reprises à la suite de l'identification d'un second comportement (étape 3) constituant le deuxième meilleur indice pour l'élève de la cote qu'il croyait que son éducateur physique lui donnerait pour sa performance en éducation physique. À cette seconde occasion, l'utilisation d'un crayon de couleur rouge 


\section{eJRIEPS 22 janvier 2011}

permettait de distinguer, sans risque de confusion, les chaînes de réponses (comportement-sentiment-réaction) pour chacun des comportements. Par la suite, l'élève devait réaliser la même procédure afin de répondre aux questions pour les deux autres dimensions de l'étude (discipline, effort).

Pour répondre aux questions relatives à une dimension, les élèves devaient obligatoirement choisir deux comportements d'éducateurs physiques différents. Toutefois, ces comportements pouvaient à nouveau être sélectionnés lors de leurs réponses aux autres dimensions. Par ailleurs, les sentiments et les réactions pouvaient être répétés plus d'une fois au sein d'une même dimension et de plusieurs dimensions du questionnaire. Au terme de cette procédure, les élèves avaient normalement identifié six comportements d'éducateurs physiques, six sentiments et six réactions. En quelques occasions, des élèves ont été autorisés à identifier un seul comportement d'éducateur physique pour une ou plusieurs dimensions compte tenu qu'ils étaient incapables d'en identifier un second.

Tableau I'. Illustration du questionnaire sur l'auto-évaluation des élèves et leur prédiction de l'évaluation de l'éducateur physique à leur égard pour la dimension Performance?

\begin{tabular}{|c|c|c|c|c|c|c|c|c|c|}
\hline \multicolumn{5}{|c|}{ 1.1 En éducation physique, tu es... } & \multicolumn{5}{|c|}{$\begin{array}{l}\text { 1.2 Que crois-tu que ton professeur d'éducation physique } \\
\text { dirait si on lui posait la même question à ton sujet? } \\
\text { II ou elle dirait que tu es... }\end{array}$} \\
\hline 1 & 2 & 3 & 4 & 5 & 1 & 2 & 3 & 4 & 5 \\
\hline \multicolumn{3}{|c|}{ Pas très bon(ne) } & \multicolumn{2}{|c|}{ Très bon(ne) } & Pas très & & & \multicolumn{2}{|c|}{ Très bon(ne) } \\
\hline
\end{tabular}




\section{eJRIEPS 22 janvier 2011}

2.1 Quels sont les comportements de ton professeur d'éducation physique qui te font croire cela ?

Mon professeur d'éducation physique...

1- me choisit pour faire des démonstrations

11- ne me fait pas confiance

2- ne me laisse pas beaucoup de chance

12- ne me choisit pas pour faire des démonstrations

3- est plus sévère avec moi

4- accepte les suggestions que je lui fais

13- ne m'encourage pas beaucoup

5- me félicite souvent

14- me choisit pour être chef d'équipe

6- ne me choisit pas pour être chef d'équipe

15- n'écoute pas ce que je lui dis

7- me donne de bonnes notes

16- me chicane rarement

8- m'accorde peu d'attention

17- m'accorde beaucoup d'attention

9- me punit souvent pour rien

18- me donne de mauvaises notes

10- me fait jouer plus souvent que d'autres élèves

19- m'encourage souvent

20- me punit rarement

Autre comportement :

2.2 Qu'est-ce que cela te fait?
1- Cela me rend malheureux (se)
2- Cela me gêne
3- Cela me décourage
4- Je suis fier (fière) de moi
5- Je me sens rejeté(e)
6- Je n'aime pas ça
7- Je suis content(e)
8- Cela me fait de la peine
9- Cela me frustre
10- Je me sens meilleur(e)
11- Cela ne me fait rien
12- Cela m'encourage
13- J'aime cela
14- Je me sens plus confiant(e)
15- Cela me choque
16- Cela me rend heureux (se)
17- Je me sens mal

17- Je me sens mal

Autre sentiment :
2.3 Comment réagis-tu lorsque cela se produit ?

1- Je me force moins

2- Je suis plus attentif (ve)

3- Je boude

4- Je travaille mieux

5- Je suis moins attentif (ve)

6- Je m'améliore

7- Je participe moins au cours

8- Je suis plus tannant(e)

9- Je continue de fournir les mêmes efforts

10- Je ne fais plus rien

11- Je me force plus

12- Je suis impoli(e)

13- Je ne respecte plus les règles

14- Je suis moins tannant(e)

15- Je travaille moins bien

16- Je participe mieux au cours

17- Je râle

18- Je continue de bien me conduire

Autre réaction : 


\section{eJRIEPS 22 janvier 2011}

${ }^{1}$ Le format du tableau a été modifié pour la revue eJRIEPS.

2 Les échelles des variables Discipline et Effort étaient respectivement libellées de Peu discipliné(e) à Très discipliné(e) et de Peu d'efforts à Beaucoup d'efforts. Les listes des comportements de l'éducateur physique, des sentiments et des réactions étaient identiques à celles utilisées pour la variable Performance.

Enfin, il importe de rappeler que les élèves avaient l'opportunité d'indiquer un comportement d'enseignant, un sentiment ou une réaction non formulés dans l'une ou l'autre des listes du questionnaire. Peu d'entre eux se sont prévalus de cette opportunité. En fait, seulement 15 des 1674 comportements d'éducateurs physiques identifiés n'étaient pas contenus dans la liste fournie aux élèves. Pour le même nombre de sentiments et de réactions d'élèves, seulement trois sentiments et trois réactions n'étaient pas déjà intégrées aux listes, confirmant ainsi la saturation des trois listes fournies aux élèves.

Dans le cadre de cet article, les éléments du questionnaire retenus sont: a) l'autoévaluation des élèves de leur performance, de leur discipline et de leurs efforts en éducation physique (Question1.1); (b) leur prédiction de l'évaluation de l'éducateur physique à leur égard pour ces mêmes trois dimensions (Question 1.2); (c) les réactions qu'ils disent adopter suite aux comportements de l'éducateur physique leur révélant la cote qu'il croyait que leur éducateur physique leur donnerait pour chacune des dimensions (Question 2.3).

\section{4. Variables considérées}

\section{4. 1. Scores de discordance}

Un score de discordance a été calculé entre la prédiction par l'élève de l'évaluation de l'éducateur physique à son égard (Question 1.2) et son auto-évaluation (Question 1.1) pour chacune des dimensions (performance, discipline, effort) considérée individuellement. Ce score de discordance permet de déterminer l'écart (ou l'absence d'écart) entre ce qu'un élève pense de lui-même et ce qu'il croit que l'éducateur physique pense de lui. L'absence d'écart signifie qu'un élève s'estime évalué à sa juste valeur alors qu'un écart, positif ou négatif, indique qu'un élève s'estime surévalué ou sous-évalué par son éducateur physique. Ces scores de discordance ont été distribués sur une échelle d'appréciation à 9 points variant entre -4 (score de discordance négative maximal) et +4 (score de discordance positif maximal), où le score zéro (0) indique une concordance (absence de discordance) entre la prédiction par l'élève de l'évaluation de l'éducateur physique à son égard et son auto-évaluation. 


\section{eJRIEPS 22 janvier 2011}

\section{4. 2. Profils de discordance}

Des profils de discordance ont été utilisés plutôt que des scores composés ( $\sum$ score de discordance performance + score de discordance discipline + score de discordance effort) afin de considérer les dimensions (performance, discipline, effort) de manière simultanée. Cette décision des chercheurs est fondée sur le fait qu'un score composé peut masquer la réalité exprimée par un élève. À titre d'exemple, un score composé de zéro, indiquant qu'un élève s'estime évalué à sa juste valeur, peut provenir de scores de discordances [($1)+(-1)+(+2)=0$ ] où l'élève se croit sous-évalué sur deux dimensions et surévalué sur une dimension.

L'utilisation de profils de discordance permet de considérer l'ensemble des tendances à la discordance des élèves eu égard à leur auto-évaluation par rapport à leur perception de l'évaluation de leur enseignant. Dix profils ont été identifiés, à l'aide de symboles, et sont présentés dans le Tableau II. Le symbole «- » représente une discordance négative (perception d'une sous-évaluation) pour l'une ou l'autre des trois dimensions, alors que le symbole « + » représente une discordance positive (perception d'une surévaluation), le symbole « $\mathbf{0}$ » signifiant une concordance (perception d'une évaluation juste). Par exemple, le profil No $5(-0+)$ signifie qu'un élève se sent sous-évalué sur une dimension, correctement évalué sur une deuxième et surévalué sur la troisième.

Tableau II. Profils de discordance.

\begin{tabular}{l|lcl|l|lll}
\hline $\begin{array}{l}\text { No de } \\
\text { profil }\end{array}$ & $\begin{array}{l}\text { Amalgames de discordances } \\
\text { pour les trois dimensions }\end{array}$ & $\begin{array}{l}\text { No de } \\
\text { profil }\end{array}$ & \multicolumn{2}{|l}{$\begin{array}{l}\text { Amalgames de discordances* } \\
\text { pour les trois dimensions }\end{array}$} \\
\hline 1 & - & - & - & 6 & - & + & + \\
2 & - & - & 0 & 7 & $\mathbf{0}$ & $\mathbf{0}$ & $\mathbf{0}$ \\
3 & - & - & + & 8 & $\mathbf{0}$ & $\mathbf{0}$ & + \\
4 & - & $\mathbf{0}$ & $\mathbf{0}$ & 9 & $\mathbf{0}$ & + & + \\
5 & - & $\mathbf{0}$ & + & 10 & + & + & + \\
\hline
\end{tabular}

${ }^{*}$ Discordance négative (-); discordance positive (+); concordance (0).

2. 4. 3. Réactions positives des élèves

Le nombre de réactions positives, basé sur les réactions que disent adopter les élèves en réaction aux comportements d'éducateurs physiques sélectionnés (Question 2.3), a été calculé pour chacune des dimensions et pour les dimensions considérées simultanément. 


\section{eJRIEPS 22 janvier 2011}

Le nombre de réactions positives varie entre zéro et deux pour chacune des dimensions et entre zéro et six pour les dimensions considérées simultanément.

\section{5. Analyse des données}

Les données descriptives usuelles ( $n$, moyennes et écart-type) ont d'abord été comptabilisées pour le nombre de réactions positives des élèves selon chacun des profils de discordance. Cette procédure a permis d'exclure d'analyses subséquentes des profils de discordance comportant un trop petit nombre de sujets (voir notes aux Tableaux III et IV). Par la suite, des analyses de variance ont permis d'examiner la relation entre les profils de discordance et le genre des élèves, puis entre les profils de discordance et le nombre de réactions positives des élèves. Des tests de comparaisons multiples (Bonferroni, $p<, 05$ ) ont également été conduits pour des valeurs $\mathrm{F}$ significatives.

\section{Résultats et discussion}

3. 1. Les profils de discordance

Le premier objectif de l'étude était de déterminer les profils de discordance d'élèves à partir de leur auto-évaluation et de leur prédiction de l'évaluation de l'enseignant à leur égard. Les Tableaux III et IV présentent les statistiques descriptives des dix profils de discordance respectivement pour les garçons et les filles et les scores de discordance composés (cumul des discordances pour les dimensions performance, discipline et effort). Les résultats révèlent qu'un peu plus de $38 \%$ des élèves (garçons=166 ; filles=154) expriment une concordance parfaite (profil 000) entre leur auto-évaluation et leur prédiction de l'évaluation de l'éducateur physique. Cela signifie que ces élèves s'estiment évalués à leur juste valeur à la fois pour leur niveau de performance, leur conduite au gymnase et leurs efforts en éducation physique. Ce pourcentage est légèrement plus élevé que celui rapporté par Julien (2003) et par Pelletier-Murphy (2004). De plus, près de 49\% des élèves $(n=406)$, garçons et filles confondus, s'estiment sous-évalués (discordance négative) sur une seule dimension $(27,7 \%)$, deux $(14,9 \%)$ ou trois dimensions $(6,1 \%)$ simultanément. Bien que les garçons soient proportionnellement plus nombreux $(50,1 \%)$ que les filles $(46,7 \%)$ à présenter une ou plusieurs discordances négatives, il n'y a pas de différence significative entre les deux distributions de profil $\left(X^{2}=11.79(6), p>.05\right)$.

Toutefois, les résultats du Tableau 5 révèlent une proportion plus élevée de discordances négatives chez les garçons pour la performance (garçons=25,8\%; filles=18,8\%) et la discipline (garçons=24,6\%; filles=14,6\%) lorsque ces dimensions sont considérées séparément. Pour la dimension effort, les garçons $(33,1 \%)$ et les filles $(33,3 \%)$ présentent 


\section{eJRIEPS 22 janvier 2011}

des proportions similaires. Sur l'ensemble des trois dimensions, 30 discordances négatives supérieures à |2| ont été observées chez les garçons et seulement trois chez les filles.

3. 2. Les profils de discordance et les comportements des élèves Le deuxième objectif de l'étude était d'analyser la relation entre les profils de discordance des élèves et les comportements qu'ils disent adopter suite à leur choix d'un comportement d'enseignant et l'identification d'un sentiment ressenti face à ce comportement. Considérant les résultats d'études antérieures (Gagnon et al., 2005 ; Pelletier-Murphy, 2004), nous avons émis l'hypothèse que les élèves qui se sentent sousévalués par leur professeur d'éducation physique (discordance négative) rapporteraient un plus petit nombre de comportements positifs. Un examen visuel des Tableaux III et IV suggère en effet que plus il y a de dimensions pour lesquelles les élèves se sentent sousévalués, moins ils tendent à rapporter de comportements positifs. 


\section{eJRIEPS 22 janvier 2011}

Tableau III. Nombre moyen de réactions positives déclarées par les garçons selon les profils de discordance et les scores composés de discordance pour chaque profil, et écarttype correspondant.

\begin{tabular}{|c|c|c|c|c|c|c|c|}
\hline & Profil & $\mathrm{N}$ & $\begin{array}{l}\text { Nombre } \\
\text { moyen de } \\
\text { réactions } \\
\text { positives }\end{array}$ & Ecart type & $\begin{array}{l}\text { Scores } \\
\text { composés }\end{array}$ & $\begin{array}{l}\text { Nombre moyen de } \\
\text { réactions } \\
\text { positives par score } \\
\text { composé }\end{array}$ & $\begin{array}{l}\text { Ecart- } \\
\text { type }\end{array}$ \\
\hline$\#$ & Type & & & & & & \\
\hline 1 & -- & 37 & 2,57 & 2,51 & -10 & 0,33 & 0,58 \\
\hline & & & & & -9 & 2,00 & --- \\
\hline & & & & & -8 & 0 & 0 \\
\hline & & & & & -6 & 0,83 & 1,17 \\
\hline & & & & & -5 & 4,00 & 1,41 \\
\hline & & & & & -4 & 2,75 & 2,87 \\
\hline & & & & & -3 & 3,80 & 2,48 \\
\hline 2 & --0 & 67 & 3,96 & 2,27 & -8 & 0 & --- \\
\hline & & & & & -6 & 3,00 & 1,41 \\
\hline & & & & & -5 & 3,00 & --- \\
\hline & & & & & -4 & 2,17 & 1,94 \\
\hline & & & & & -3 & 3,00 & 2,67 \\
\hline & & & & & -2 & 4,47 & 2,09 \\
\hline \multirow[t]{4}{*}{4} & -00 & 94 & 4,84 & 1,80 & -4 & 3,00 & --- \\
\hline & & & & & -3 & 4,00 & --- \\
\hline & & & & & -2 & 3,67 & 2,94 \\
\hline & & & & & -1 & 4,95 & 1,70 \\
\hline 7 & 000 & 166 & 5,61 & 0,91 & 0 & 5,61 & 0,91 \\
\hline \multirow[t]{3}{*}{8} & $00+$ & 46 & 5,43 & 1,13 & +1 & 5,55 & 0,92 \\
\hline & & & & & +2 & 3,67 & 2,52 \\
\hline & & & & & +4 & 6,00 & --- \\
\hline 9 & $0++$ & 8 & 5,50 & 0,76 & +2 & 5,50 & 0,76 \\
\hline \multirow[t]{2}{*}{10} & +++ & 3 & 4,00 & 1,00 & +3 & 4,00 & 1,41 \\
\hline & & & & & +6 & 4,00 & --- \\
\hline 3 & --+ & 8 & 2,38 & 1,92 & --- & 2,38 & 1,92 \\
\hline 5 & $-0+$ & 15 & 4,80 & 1,57 & --- & 4,80 & 1,57 \\
\hline 6 & -++ & 3 & 3,67 & 2,52 & --- & 3,67 & 2,52 \\
\hline
\end{tabular}




\section{eJRIEPS 22 janvier 2011}

$1 \mathrm{~N}$ total $=447$ garçons (26 des 473 garçons avaient identifié un seul comportement d'enseignant pour une ou plusieurs des dimensions)

${ }^{2}$ Les profils 3, 6 et 10 ont été écartés des analyses ultérieures en raison du nombre réduit de sujets dans ces catégories.

Tableau IV. Nombre moyen de réactions positives déclarées par les filles selon les profils de discordance et les scores composés de discordance pour chaque profil, et écart-type correspondant.

\begin{tabular}{|c|c|c|c|c|c|c|c|}
\hline & Profil & $\mathrm{N}$ & $\begin{array}{l}\text { Nombre } \\
\text { moyen de } \\
\text { réactions } \\
\text { positives }\end{array}$ & Ecart type & $\begin{array}{l}\text { Scores } \\
\text { composés }\end{array}$ & $\begin{array}{l}\text { Nombre moyen de } \\
\text { réactions } \\
\text { positives par score } \\
\text { composé }\end{array}$ & $\begin{array}{l}\text { Ecart- } \\
\text { type }\end{array}$ \\
\hline$\#$ & Type & & & & & & \\
\hline \multirow[t]{4}{*}{1} & --- & 14 & 3,00 & 2,04 & -6 & 3,25 & 2,75 \\
\hline & & & & & -5 & 1,50 & 2,12 \\
\hline & & & & & -4 & 3,00 & --- \\
\hline & & & & & -3 & 3,29 & 1,89 \\
\hline \multirow[t]{3}{*}{2} & --0 & 49 & 4,47 & 1,86 & -4 & 4,00 & --- \\
\hline & & & & & -3 & 3,83 & 1,72 \\
\hline & & & & & -2 & 4,57 & 1,90 \\
\hline \multirow[t]{2}{*}{4} & -00 & 94 & 5,27 & 1,28 & -2 & 5,50 & 0,71 \\
\hline & & & & & -1 & 5,26 & 1,29 \\
\hline 7 & 000 & 154 & 5,77 & 0,61 & 0 & 5,77 & 0,61 \\
\hline \multirow[t]{2}{*}{8} & $00+$ & 41 & 5,63 & 1,07 & +1 & 5,63 & 1,08 \\
\hline & & & & & +4 & 6,00 & --- \\
\hline 9 & $0_{++}$ & 9 & 5,67 & 0,50 & +2 & 5,67 & 0,50 \\
\hline \multirow[t]{2}{*}{10} & +++ & 4 & 3,25 & 3,20 & +3 & 6,00 & 0 \\
\hline & & & & & +5 & 0,50 & 0,71 \\
\hline 3 & --+ & 1 & 4,00 & --- & --- & 4,00 & --- \\
\hline 5 & $-0+$ & 21 & 5,33 & 1,02 & --- & 5,33 & 1,02 \\
\hline 6 & -++ & 3 & 4,00 & 3,46 & --- & 4,00 & 3,46 \\
\hline
\end{tabular}




\section{eJRIEPS 22 janvier 2011}

${ }^{2}$ Les profils 3,6 et 10 ont été écartés des analyses ultérieures en raison du nombre réduit de sujets dans ces catégories.

Tableau V. Amplitude et distribution relative des scores de discordance chez les garçons et les filles en fonction de chacune des trois dimensions.

\begin{tabular}{|c|c|c|c|c|c|c|c|c|c|c|c|c|}
\hline \multirow{3}{*}{ Amplitude } & \multicolumn{4}{|c|}{ Performance } & \multicolumn{4}{|c|}{ Discipline } & \multicolumn{4}{|c|}{ Effort } \\
\hline & \multicolumn{2}{|c|}{ Filles } & \multicolumn{2}{|c|}{ Garçons } & \multicolumn{2}{|c|}{ Filles } & \multicolumn{2}{|c|}{ Garçons } & \multicolumn{2}{|c|}{ Filles } & \multicolumn{2}{|c|}{ Garçons } \\
\hline & $\mathrm{N}$ & $\%$ & $\mathrm{~N}$ & $\%$ & $\mathrm{~N}$ & $\%$ & $\mathrm{~N}$ & $\%$ & $\mathrm{~N}$ & $\%$ & $\mathrm{~N}$ & $\%$ \\
\hline \multicolumn{13}{|c|}{ Discordances } \\
\hline \multicolumn{13}{|l|}{ négatives } \\
\hline-4 & --- & --- & 2 & 0,5 & --- & --- & 4 & 0,9 & --- & --- & 9 & 2,0 \\
\hline-3 & --- & --- & 7 & 1,6 & --- & --- & 2 & 0,4 & 3 & 0,7 & 6 & 1,3 \\
\hline-2 & 10 & 2,6 & 20 & 4,5 & 4 & 1,0 & 11 & 2,5 & 8 & 2,1 & 19 & 4,3 \\
\hline-1 & 63 & 16,2 & 86 & 19,2 & 53 & 13,6 & 93 & 20,8 & 119 & 30,5 & 114 & 25,5 \\
\hline Sous-total & 73 & 18,8 & 115 & 25,8 & 57 & 14,6 & 110 & 24,6 & 130 & 33,3 & 148 & 33,1 \\
\hline \multicolumn{13}{|c|}{ Concordance } \\
\hline 0 & 268 & 68,6 & 303 & 67,7 & 305 & 78,2 & 296 & 66,2 & 238 & 61,0 & 269 & 60,2 \\
\hline \multicolumn{13}{|c|}{ Discordances } \\
\hline \multicolumn{13}{|l|}{ positives } \\
\hline+1 & 49 & 12,6 & 27 & 6,0 & 25 & 6,4 & 37 & 8,3 & 18 & 4,6 & 26 & 5,8 \\
\hline+2 & --- & --- & 2 & 0,5 & 3 & 0,8 & 3 & 0,7 & 2 & 0,5 & 2 & 0,5 \\
\hline+3 & --- & --- & --- & --- & --- & --- & 1 & 0,2 & 1 & 0,3 & 1 & 0,2 \\
\hline+4 & --- & --- & --- & --- & --- & --- & --- & --- & 1 & 0,3 & 1 & 0,2 \\
\hline Sous-total & 49 & 12,6 & 29 & 6,5 & 28 & 7,2 & 41 & 9,2 & 22 & 5,7 & 30 & 6,7 \\
\hline Total & 390 & 100 & 447 & 100 & 390 & 100 & 447 & 100 & 390 & 100 & 447 & 100 \\
\hline
\end{tabular}

Nous avons effectué une analyse de variance à deux dimensions en prenant le sexe et le profil de discordance comme variable indépendante et le nombre de comportements positifs rapportés comme variable dépendante. Des différences significatives ont été observées pour le sexe $[F=5.21(1), p .=.023]$ et pour le profil de discordance $[F=36.56$ (6), p. = .000] (Figure 2). Quel que soit le profil de discordance, les garçons rapportent un score total de comportements positifs plus faible. La Figure 2 présente également le résultat d'un test de comparaisons multiples (Bonferroni), à un niveau de signification de 0,05 , pour les profils de discordance, garçons et filles confondus. Selon les règles d'édition usuelles, les valeurs moyennes reliées par un trait ne présentent pas de différence 


\section{eJRIEPS 22 janvier 2011}

significative entre elles. Le test confirme notre constat initial pour les cas où le profil présente au moins deux signes négatifs, indiquant par là que ces élèves se percevaient sous-évalués par leur enseignant sur deux ou trois dimensions (les valeurs 4.17 et 2.69 sont significativement différentes l'une de l'autre et sont également significativement différentes de toutes les autres).

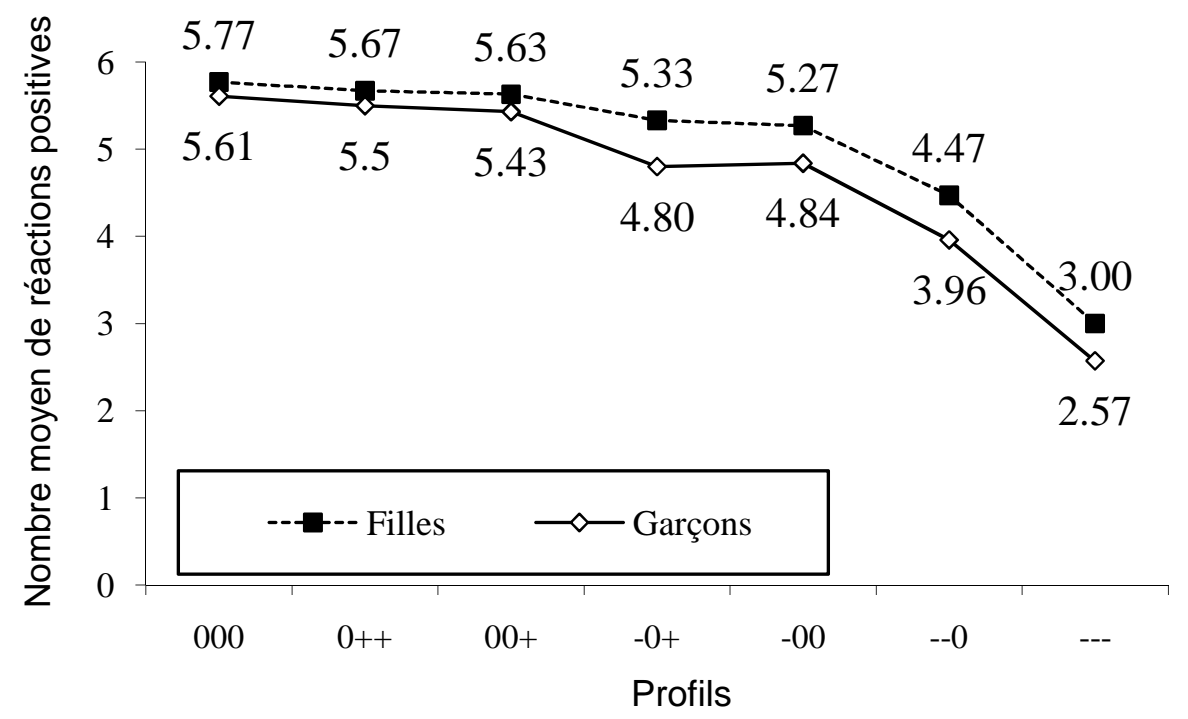

Test de comparaisons multiples (garçons et filles confondus)

Profils

$000 \quad 0++\quad 00+\quad-0+\quad-00 \quad--0$

Nombre moyen de réactions positives

Figure 2. Nombre moyen de réactions positives rapportées par les garçons et les filles pour chaque profil de discordance et test de comparaisons multiples (garçons et filles confondus)

3. 3. Les scores de discordance et les comportements des élèves

Le troisième objectif de la recherche était de déterminer s'il y a une relation, pour chacune des trois dimensions étudiées, entre l'amplitude de la discordance perçue par les élèves et les comportements qu'ils disent adopter durant les leçons d'éducation physique. Les amplitudes des scores de discordance pour garçons et filles en fonction de chacune des trois dimensions sont présentées au Tableau V. La prédominance de concordance (absence de discordance) est notable chez les garçons et les filles et au niveau des trois 


\section{eJRIEPS 22 janvier 2011}

dimensions. Pour les discordances, les garçons sont plus nombreux sur les scores de discordances élevés, particulièrement négatifs.

Le nombre de réactions positives associées aux divers niveaux de discordance pour les dimensions (performance, discipline, effort) considérées séparément est présenté à la Figure 3. Nous avons choisi d'utiliser uniquement les valeurs se situant entre -2 et +2 (plutôt que celles entre -4 et +4 ) dans la mesure où très peu de filles présentent des scores de discordance (positifs ou négatifs) supérieurs à | 2 | dans chaque dimension (voir Tableau V). Tel que confirmé par des analyses de variance, plus les élèves se perçoivent sous-évalués par leur enseignant, que ce soit pour la performance, la discipline ou l'effort, moins ils tendent à adopter des comportements positifs en réaction aux comportements de leur enseignant [performance $: F=42,39(3), p .=0,000$; discipline $: F=37,42(3)$, p. $=$ 0,000 ; effort : $F=23,13(3)$, p. = 0,000]. Pour garçons et filles confondus, les résultats du test de comparaisons multiples de Bonferroni (niveau alpha $=0,05$ ) pour chaque dimension sont indiqués sur la droite de la Figure 3. Chaque fois que les élèves se sentent sous-évalués, quelle que soit la dimension en cause, le nombre de réactions positives rapportées est plus faible. Le nombre moyen (garçons et filles ensemble) de réactions positives chute fortement lorsque la discordance négative passe de |1| à |2|. Pour la dimension performance, les résultats montrent que les filles ont signalé un nombre significativement plus élevé de réactions positives que les garçons $[F=5,52(1)$, p. = 0,019].

\section{Performance}

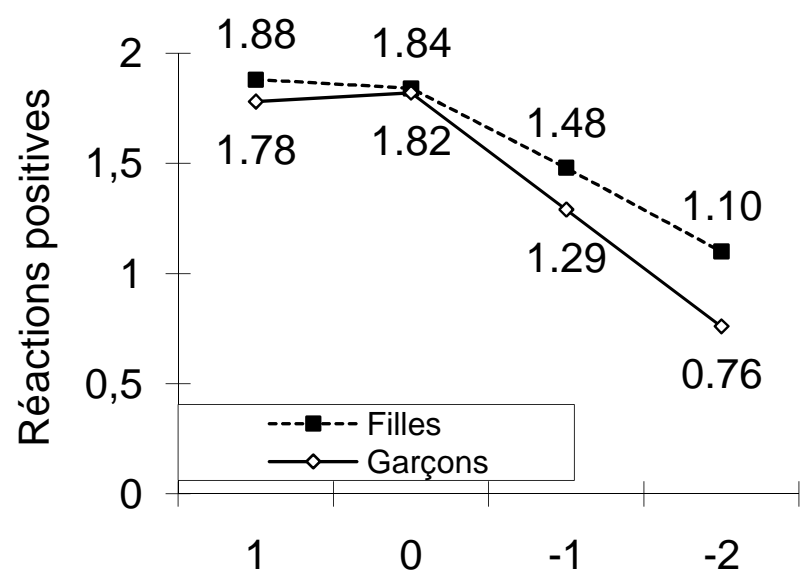

Test de comparaisons multiples

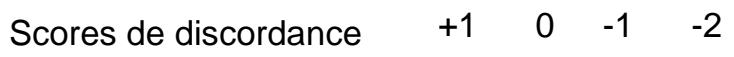




\section{Discipline}

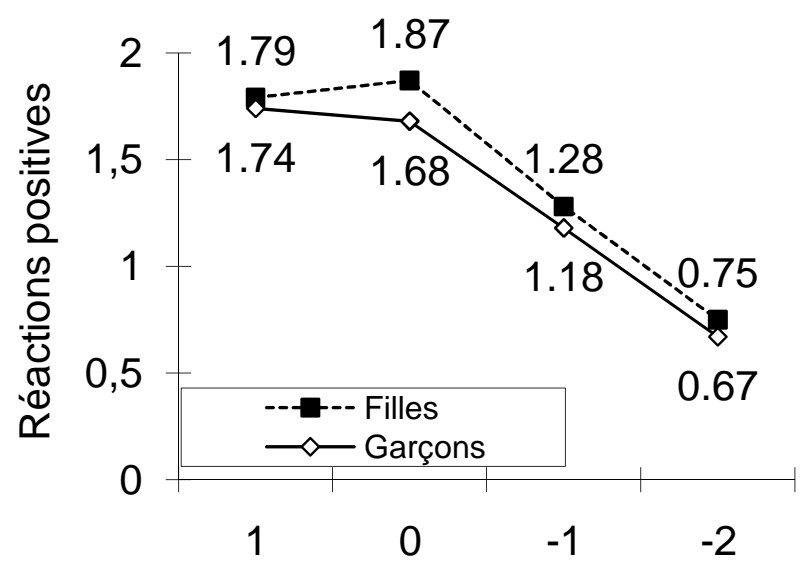

\section{Effort}

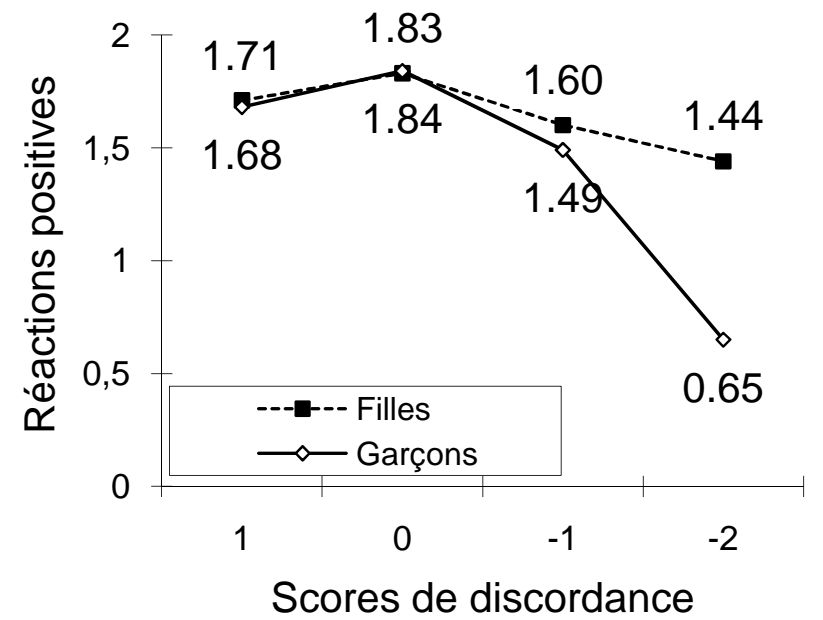

Test de comparaisons multiples

Scores de discordance

$\begin{array}{llll}0 & +1 & -1 & -2\end{array}$

Réactions positives

$\begin{array}{llll}1.78 & 1.76 & 1.21 & 0.69\end{array}$
Test de comparaisons multiples

Scores de discordance

Réactions positives

$$
\begin{array}{cccc}
0 & +1 & -1 & -2 \\
1.84 & 1.69 & 1.55 & 0.90 \\
\hline
\end{array}
$$

Figure 3. Nombre de réactions positives rapportées par les garçons et les filles en fonction des scores de discordance pour chaque dimension (Performance, Discipline, Effort) et test de comparaisons multiples (garçons et filles confondus).

Dans la section effort, les garçons qui présentent la plus forte discordance négative retenue (-2) ont signalé un nombre de réactions positives encore plus faible que celui des filles. L'interaction «score de discordance / sexe» $[F=3,69(3), p .=0,012]$ est significative. 


\section{eJRIEPS 22 janvier 2011}

\section{Conclusion et pistes de recherche}

Les résultats montrent que les perceptions qu'ont les élèves de ce que leur enseignant pense d'eux en termes de performance, de discipline et d'effort, ont un impact sur les comportements qu'ils disent adopter en classes d'éducation physique. D'une part, plus des élèves perçoivent être en accord avec leur enseignant, plus ils disent adopter des comportements positifs. D'autre part, lorsque des élèves se sentent sous-évalués, ils tendent à signaler moins de comportements positifs. Cela semble être particulièrement le cas lorsque les élèves se sentent sous-évalués sur au moins deux des trois dimensions étudiées. Si l'on considère chaque dimension séparément, il semble également que des discordances négatives supérieures à $|1|$ sont associées à un nombre plus élevé de comportements négatifs. De plus, l'étude a démontré que les garçons ont davantage tendance à se sentir sous-évalués et à présenter dans ce cas des discordances négatives supérieures à $|2|$. II en ressort donc que les garçons, en moyenne, rapportent moins de comportements positifs que les filles.

4. 1. La valeur du concept de discordance

L'étude a permis de démontrer que la nature et l'amplitude de l'écart entre l'autoévaluation d'un élève et sa prédiction de l'évaluation de l'éducateur physique constitue un indice des comportements qu'il est susceptible d'adopter en éducation physique. Ces comportements, associés à la qualité de son engagement et des efforts consentis lors des cours d'ÉPS, peuvent faire en sorte de favoriser ou de nuire à son développement et à ses apprentissages. Par conséquent, la discordance entre l'auto-évaluation d'un élève et sa perception de l'évaluation de son enseignant, que ces dernières soient justes ou erronées, semble être une variable pertinente pour l'étude du processus enseignementapprentissage.

\section{2. Pistes de recherche}

II nous apparaît essentiel de réaliser des recherches qui permettent de poursuivre l'étude de la relation entre la nature et l'amplitude de l'écart entre l'auto-évaluation d'élèves et leur prédiction de l'évaluation de l'éducateur physique (discordance) et les comportements qu'ils disent adopter en ÉPS. Par exemple, ces recherches pourraient être réalisées auprès d'élèves d'âges variés (niveau primaire, niveau secondaire), au sein de classes mixtes et non mixtes en ÉPS ainsi que dans différents pays. À ce titre, les résultats d'une recherche réalisée récemment en Belgique, auprès d'élèves de l'enseignement général au secondaire, sont en cours d'analyse. Enfin, il pourrait être également intéressant d'utiliser le concept de discordance pour des études en contextes sportifs auprès d'athlètes d'âges 


\section{eJRIEPS 22 janvier 2011}

variés qui évoluent dans des disciplines sportives individuelles ou collectives à des niveaux de compétition différenciés.

Par ailleurs, il nous apparaît nécessaire de décrire le processus enseignementapprentissage dans des classes où l'on observe soit peu, soit beaucoup de discordances entre ce que les élèves pensent d'eux-mêmes et ce qu'ils ont l'impression que leur éducateur physique pense d'eux. Ainsi, nous pourrions tenter de déterminer ce qui peut provoquer de telles discordances entre ce que les élèves pensent d'eux-mêmes et ce qu'ils ont l'impression que leur enseignant pense d'eux. II serait également pertinent de documenter ce que vivent des élèves qui expriment de fortes discordances. Une meilleure compréhension de leur réalité pourrait constituer le point de départ de recherches-action contribuant à réduire la fréquence de ces discordances.

Enfin, d'autres recherches-action pourraient amener élèves et enseignants à reconsidérer leurs perceptions quand celles-ci s'avèrent être plus ou moins justes par rapport à la réalité. Plus précisément, les élèves peuvent se tromper dans leur auto-évaluation et les enseignants peuvent de fait sous-estimer ou surévaluer leurs élèves. Dans de tels cas, il semble utile de mettre en place un processus de régulation des perceptions fondé sur des données tirées de l'observation systématique des comportements des élèves durant le processus enseignement-apprentissage. Une prise de conscience des comportements réels des élèves pourrait permettre à la fois aux élèves et aux enseignant de réajuster, au besoin, leurs perceptions. À cet effet, le profil des comportements effectifs des élèves pourrait être dressé à l'aide de systèmes d'observation servant à déterminer leur niveau de réussite dans les tâches d'apprentissage (performance), la fréquence et gravité des comportements déviants qu'ils adoptent (discipline), et l'intensité des efforts qu'ils démontrent lors de la réalisation des tâches en classes d'éducation physique.

Pour terminer, il nous importe de préciser que nous croyons qu'il revient aux enseignants de tenter de corriger des conditions d'enseignement-apprentissage qui semblent nuire au développement de leurs élèves. Sanders (1996) et Tjeerdsma (1997) ont exprimé un point de vue similaire dans leurs publications destinées à aider les enseignants d'éducation physique à mieux comprendre les expériences d'éducation physique de leurs élèves. De plus, il convient sans doute de rappeler aux enseignants et chercheurs les mots de Graham (1995) :

« L'un des messages les plus puissants de cette monographie est le rappel poignant que les élèves, mêmes ceux aussi jeunes que cinq ans, sont capables d'exprimer leurs sentiments, leurs besoins et leur pensées par 


\section{eJRIEPS 22 janvier 2011}

rapport à ce qu'on leur enseigne en éducation physique et à la façon dont c'est enseigné ». (p. 481, traduction libre)

\section{Bibliographie}

Babad, E.Y., Bernieri,F., \& Rosenthal, R.A. (1991). Students as judges of teachers' verbal and nonverbal behavior. American Educational Research Journal, 28, 211-234.

Brattesani, K. A., Weinstein, R. S., \& Marshall, H. H. (1984). Student perceptions of differential teacher treatment as moderators of teacher expectation effects. Journal of Educational Psychology, 76, 236-247.

Brophy, J.E. (1983). Research on the self-fulfilling prophecy and teacher expectations. Journal of Educational Psychology, 75, 631-661.

Daniels, R. (1983). The development of a tool to measure perceptions of physical education teaching behaviors found in secondary schools. Unpublished doctoral dissertation, University of North Carolina at Greensboro.

Dostie, S. (1996). Analyse d'incidents disciplinaires vécus par des éducateurs physiques au primaire. Mémoire de Maîtrise, Université du Québec à Trois-Rivières, TroisRivières, Québec, Canada.

Dyson, B.P. (1995). Students' voices in to alternatives elementary physical education programs. Journal of Teaching in Physical Education, 14, 394-407.

Fraser, B.J. (1994). Student Perceptions of Classrooms. In T. Husen, \& T.N. Postlethwaite (Eds.), The international encyclopedia of education (pp. 5772-5775). New York: Pergamon.

Gagnon, J. (1992). L'effet Pygmalion dans une équipe sportive de niveau collégial. Thèse de Doctorat, Université Laval, Québec, Canada.

Gagnon, J., Martel, D., Michaud, V., Valois. P., \& Gagné, G. (2005). Types de concordance-discordances entre l'auto-évaluation des élèves et leur prédiction de l'évaluation de leur éducateur physique. Revue de l'Éducation Physique, 45, 79-90.

Good, T.L. (1987). Two decades of research on teacher expectations: Findings and future directions. Journal of Teacher Education, 38(4), 32-47.

Graham, G. (1995). Physical education through students' eyes and in students' voices: Implications for teachers and researchers. Journal of Teaching in Physical Education, 14, 478-482.

Halliburton, A.L., \& Weiss, M.R. (2002). Sources of competence information and perceived motivational climate among adolescent female gymnasts varying in skill level. Journal of Sport and Exercise Psychology, 24, 396-419. 


\section{eJRIEPS 22 janvier 2011}

Julien, A. (2003). Étude de la concordance entre ce que des élèves pensent d'eux-mêmes et ce qu'ils croient que leur éducateur physique pense d'eux. Mémoire de Maîtrise, Université du Québec à Trois-Rivières, Trois-Rivières, Québec, Canada.

Lee, A.M., \& Solmon, M.A. (2005). Pedagogy research through the years in RQES. The Research Quarterly for Exercise and Sport, 76(Supplement to number 2), S108S121.

Lentillon, V. (2005). Privation, justice, inégalités: Perceptions différenciées chez les filles et les garçons au niveau des notes et du soutien obtenus en EPS ? Dans G. Cogérino, (Ed.), Filles et garçons en EPS (pp. 181-197). Paris (France): Éditions Revue EP.S.

Martel, D., Gagnon, J., Grenier, J., Pelletier-Murphy, J., \& Dumont, S. (1999). Révélations d'élèves du primaire sur la conduite de l'éducateur physique à leur égard. Dans J.F. Gréhaigne, N. Mahut, \& D. Marchal (Eds.), Qu'apprennent les élèves en faisant des activités physiques et sportives? - What do people learn from physical activity programs? Actes du Congrès de l'Association internationale des écoles supérieures d'éducation physique [CD-ROM]. Besançon, France: IUFM de Franche-Comté.

Martel, D., Gagnon, J., Pelletier-Murphy, J., \& Grenier, J. (1999). Pygmalion en éducation physique : Un mythe bien réel. Revue Canadienne de l'Éducation, 24, 42-56.

Martel, D., Pelletier-Murphy, J., \& Gagnon, J. (1999, avril). Perceptions d'élèves sur la façon dont ils sont traités en éducation physique. Présentation sur affiche au congrès annuel de l'AIESEP, Besançon, France.

Martinek, T.J. (1981). Physical attractiveness : Effects on teacher expectations and dyadic interactions in elementary age children. Journal of Sport Psychology, 3, 196-205.

Martinek, T.J. (1988). Confirmation of a teacher expectancy model: Student perceptions and causal attributions of teaching behaviors. Research Quarterly for Exercise and Sport, 59, 118-126.

Martinek, T. J. (1991). Psycho-social dynamics of teaching physical education. William C. Brown Pub.

Martinek, T.J., \& Karper, W. B. (1986). Motor ability and instructional contexts: Effect on teacher expectations and dyadic interactions in elementary physical education classes. Journal of Classroom Interaction, 21(2), 16-25.

McKiddie, B., \& Maynard, I. W. (1997). Perceived competence of school children in physical education. Journal of Teaching in Physical Education, 16, 324-339.

Morency, L. (1990). Pygmalion dans des classes d'éducation physique au primaire [Pygmalion in elementary school PE classes]. Revue Canadienne de l'Éducation, 15, 103-114. 


\section{eJRIEPS 22 janvier 2011}

Mros, M. (1990). A description of the causal attributions made to perceived teaching behavior across three elementary physical education context. Unpublished doctoral dissertation, University of North Carolina at Greensboro.

Nicaise, V. (2005). Les feedback: Perception différenciées chez les filles et les garçons? Dans G. Cogérino, (Ed.), Filles et garçons en EPS (pp. 199-219). Paris (France): Éditions Revue EP.S.

Nicaise, V., Cogérino, G., Bois, J. E., \& Amorose, A. J. (2006). Students' perceptions of teacher feedback and physical competence in physical education classes: Gender effects. Journal of Teaching in Physical Education, 25, 36-57.

Nicaise, V., Bois, J. E., Fairclough, S. J., Amorose, A. J., \& Cogérino, G. (2007). Girls' and boys' perceptions of physical education teachers' feedback: Effects on performance and psychological responses. Journal of Sports Sciences, 25, 915-926.

Pelletier-Murphy, J. (2004). Perceptions d'élèves du primaire qui s'estiment sous-évalués, surévalués ou évalués à leur juste valeur par leur éducateur physique. Thèse de Doctorat, Université de Montréal, Québec, Canada.

Sanders, S. W. (1996). Children's physical education experiences - Their interpretations can help teachers. Journal of Physical Education, Recreation \& Dance, 67(3), 51-56.

Tjeerdsma, B. L. (1997). Enhancing classroom communication between teacher and student. Journal of Physical Education, Recreation \& Dance, 68(5), 26-28, 32.

Trouilloud, D. O. \& Sarrazin, P. G. (2003). Les connaissances actuelles sur l'effet Pygmalion : Processus, poids et modulateurs. Revue Française de Pédagogie, № 145, 89-119.

Trouilloud, D. O., Sarrazin, P. G., Martinek, T. J. \& Guillet, E. (2002). The influence of teacher expectations on student achievement in physical education classes: Pygmalion revisited. European Journal of Social Psychology, 32,591-607.

Weinstein, R.S. (1983). Student perceptions of schooling. Elementary School Journal, 38, 287-312.

Weinstein, R. S.,Marshall, H. H., Brattesani, K. A. \& Middlestadt, S. E. (1982). Student perceptions of differential teacher treatment in open and traditional classrooms. Journal of Educational Psychology, 74, 678-692.

Weinstein, R. S., Marshall, H. H., Sharp, L., \& Botkin, M. (1987). Pygmalion and the student: Age and classroom differences in children's awareness of teacher expectations. Child Development, 58, 1079-1093. 


\section{eJRIEPS 22 janvier 2011}

Weiss, M. R., \& Amorose, A. J. (2005). Children self-perceptions in the physical domain: Between- and within-age variability in level, accuracy, and sources of perceived competence. Journal of Sport and Exercise Psychology, 27, 226-244.

Weiss, M. R., Ebbeck, V., \& Horn, T. S. (1997). Children's self-perception and sources of physical competence information: A cluster analysis. Journal of Sport and Exercise Psychology, 19, 52-70. 